\title{
Lumbar Disc Degeneration is Facilitated by MiR-100-Mediated FGFR3 Suppression
}

\author{
Ning Yan Shunzhi Yu Hailong Zhang Tiesheng Hou \\ Orthopedic Department, Shanghai 10th People's Hospital, Tongji University School of Medcine, Shanghai, \\ China
}

\section{Key Words}

Lumbar disc degeneration (LDD) • Matrix metalloproteinase $13(\mathrm{MMP13}) \cdot$ MiR-100 • Fibroblast growth factor receptor 3 (FGFR3)

\begin{abstract}
Background/Aims: The pathogenesis of lumbar disc degeneration (LDD) involved activation of matrix metalloproteinase 13 (MMP13) by differential expression of fibroblast growth factor receptor 1 (FGFR1) and FGFR3. Nevertheless, the molecular regulation of FGFR1 and FGFR3 in the lumber disc cells remains elusive. Methods: We examined the FGFR1 and FGFR3 levels and microRNAs (miRNAs) levels in the resected LDD discs, compared to the traumatized, non-LDD discs. We analyzed the binding of miR-100 to the 3'UTR of FGFR3 mRNA and its effects on FGFR3 translation by bioinformatics analysis and by luciferase-reporter assay, respectively. We modified miR-100 levels in a human nucleus pulposus SV40 cell line (HNPSV), and examined the effects on the expression of FGFR3 and MMP13, by RT-qPCR, Western blot and ELISA. Results: The levels of FGFR1 and miR-100 were significantly higher, while the levels of FGFR3 were significantly lower, in LDD discs, compared to the control non-LDD discs. The levels of FGFR3, but not the levels of FGFR1, inversely correlated with the levels of miR-100. Moreover, miR-100 was found to bind to the $3^{\prime} U T R$ of FGFR3 mRNA to prevent its translation. In miR100-modified HNPSV cells, we found that miR-100 decreased FGFR3 levels, and increased MMP13 levels. Conclusion: miR-100 may activate MMP13 through 3'UTR-suppressoin of FGFR3 mRNA to facilitate development of LDD.
\end{abstract}

Copyright $\odot 2015$ S. Karger AG, Basel

\section{Introduction}

Lumbar disc degeneration (LDD) often causes severe pain on neck and low back of the body. LDD often occurs in a degenerated disc in the spine, and appears to be a product of lifelong degradation of the discs and neighboring tissue [1-3]. The diagnosis of LDD relies

Tiesheng Hou

KARGER 125
Orthopedic Department, Shanghai 10th People's Hospital, Tongji University School of Medcine, 301 Yanchang Middle Road, Shanghai 200072 (China)

Tel. +862166300588, E-Mail tiesheng_hou@163.com 


\section{Cellular Physiology Cell Physiol Biochem 2015;36:2229-2236 \begin{tabular}{l|l|l} 
DOI: 10.1159/000430187 & $\begin{array}{l}\text { O 2015 S. Karger AG, Basel } \\
\text { www.karger.com/cpb }\end{array}$ \\
\hline
\end{tabular} \\ Yan/Yu/Zhang/Hou: MiR-100 Promotes LDD through FGFR3}

on the clinical symptoms, radiographic magnetic resonance imaging, microscopic, histologic and biochemical analysis, macroscopic measurement of degeneration in surgical and autopsy samples [1-3]. Although LDD is a prevalent disease and has been extensively studies for decades, the pathogenesis remains largely undetermined.

The molecular pathogenesis of LDD has been extensively studied in the past. Disc cells actively regulate matrix homeostasis through activities modulated by a variety of cytokines and growth factors acting in a paracrine and/or autocrine fashion [4]. While normal disc cells maintain the matrix at a steady state, an imbalance between the anabolic and catabolic processes in the pathological processes result in LDD [4]. Disc damage caused by mechanical injury, inflammation, or aging may modulate the structure of the discs through regulating disc homeostasis [5]. Matrix metalloproteinase 13 (MMP13) is also called collagenase 3 [69], and has been found to strongly upregulate by proinflammatory cytokines related to LDD, in which MMP13 acts as a proteoglycans (PG)-degrading enzyme in addition to assisting in collagen degradation for promoting LDD [10]. PG is critical for disc cell maintenance and regeneration after injury $[11,12]$. The evidence of involvement of other MMPs in the development of LDD is much lacking.

MMP13 has been shown to be regulated by fibroblast growth factor 2 (FGF2) through binding to fibroblast growth factor receptor 1 (FGFR1) $[13,14]$. The FGFRs have 4 different isoforms named 1-4. These FGFRs consist of a cellular ligand domain composed of three immunoglobulin-like domains, a single transmembrane helix domain, and an intracellular domain with tyrosine kinase activity to mediate signal transduction after their binding to FGF ligands [15-17]. FGFR1 and FGFR3 compete with each other for FGF2 bindings [18, 19]. Hence, FGFR3 is suggested to have a negative effect on FGF2-mediated MMP13 expression $[18,19]$. However, the exact underlying molecular mechanisms are largely unknown.

MicroRNAs (miRNAs) are a class of small, non-coding RNAs that regulate various biological processes [20-22]. Interestingly, bioinformatics approaches have predicted onethird of all mammalian genes to be targeted and regulated by miRNAs [20-22]. However, the role of miRNAs in the development of LDD has not been reported.

Here, we reported that the levels of FGFR1 and miR-100 were significantly higher, while the levels of FGFR3 were significantly lower, in LDD discs, compared to the control non-LDD discs. The levels of FGFR3, but not the levels of FGFR1, inversely correlated with the levels of miR-100. Moreover, miR-100 was found to bind to the 3'UTR of FGFR3 mRNA to prevent its translation. In miR-100-modified HNPSV cells, we found that miR-100 decreased FGFR3 levels, and increased MMP13 levels.

\section{Materials and Methods}

\section{Patient specimens}

A total of 37 subjects (21 LDD discs and 16 traumatized, non-LDD discs) were included in this study. All the subjects had no accompanying diseases (e.g. diabetes, hepatitis) that may affect the study on FGFR levels. All specimens had been histologically and clinically diagnosed at Orthopedic Department, Shanghai 10th People's Hospital, Tongji University from 2009 to 2014. For the use of these clinical materials for research purposes, prior patient's consents and approval from the Institutional Research Ethics Committee were obtained.

Culture, transfection and treatment of human disc cells

A human disc cell line, nucleus pulposus SV40 (HNPSV) has been described before [23]. The HNPSV was a gift from Prof. Sakai, and was cultured in Dulbecco's modified Eagle medium (DMEM, Gibco; Life Technologies, Carlsbad, CA, USA) with 10\% fetal bovine serum (FBS, Sigma-Aldrich, St Louis, MO, USA), penicillin $(100 \mu \mathrm{g} / \mathrm{ml})$ and streptomycin $(250 \mathrm{ng} / \mathrm{ml})$ at $37^{\circ} \mathrm{C}$, in a $5 \% \mathrm{CO}_{2}$ incubator. HNPSV cells were transfected with a miR-100 construct, or an antisense (as) of miR-100, or a scramble sequence (scr) as a control. MiR-100 sequence: 5'- AACCCGUAGAUCCGAACUUGUG-3', miR-100 antisense sequence: 5'-CACAAGUUCGGAUCUACGGGUU-3', control sequence: 5'-UUUAGCGCCGAAAAGUAAUG-3'. Transfection was performed with Lipofectamine 2000 reagent (Invitrogen), according to the manufacturer's instructions. 


\section{Cellular Physiology Cell Physiol Biochem 2015;36:2229-2236 \begin{tabular}{l|l} 
and Biochemistry $\begin{array}{l}\text { DOI 10.1159/000430187 } \\
\text { Published online: July 24, } 2015\end{array}$ & $\begin{array}{l}\text { C 2015 S. Karger AG, Basel } \\
\text { www.karger.com/cpb }\end{array}$ \\
\hline
\end{tabular} \\ Yan/Yu/Zhang/Hou: MiR-100 Promotes LDD through FGFR3}

\section{Luciferase-reporter activity assay}

Luciferase-reporters were successfully constructed using molecular cloning technology. Target sequence was inserted into pGL3-Basic vector (Promega, Madison, WI, USA) to obtain pGL3-FGFR3-3'UTR, which contains the miR-100 binding sequence (FGFR3-3'UTR sequence). HNPSV-miR-100, or HNPSVscr, or HNPSV-antisense (as)-miR-100 cells were seeded in 24-well plates for 24 hours, after which they were transfected with $1 \mu \mathrm{g}$ of Luciferase-reporter plasmids per well using PEI Transfection Reagent. Then luciferase activities were measured using the dual-luciferase reporter gene assay kit (Promega), according to the manufacturer's instructions.

Quantitative PCR (RT-qPCR)

MiRNA and total RNA were extracted from cultured cells with miRNeasy mini kit or RNeasy kit (Qiagen, Hilden, Germany), respectively, and used for cDNA synthesis. Quantitative PCR was performed in duplicates with QuantiTect SYBR Green PCR Kit (Qiagen). All primers were purchased from Qiagen. Data were analyzed with 2- $\Delta \Delta \mathrm{Ct}$ method for quantification of the relative mRNA expression levels. Values of genes were normalized against $\alpha$-tubulin, and then compared to controls.

\section{Western blot}

The protein was extracted from the resected OS specimen or adjacent normal bone tissue (NT), or cultured cells. Primary antibodies were anti-FGFR3 and anti- $\alpha$-tubulin (Cell Signaling, San Jose, CA, USA). $\alpha$-tubulin was used as a protein loading control. Secondary antibodies were HRP-conjugated anti-rabbit, and were all purchased from Jackson ImmunoResearch Labs (West Grove, PA, USA). Densitometry of Western blots was quantified by NIH ImageJ software (Bethesda, MA, USA). The protein levels were first normalized to $\alpha$-tubulin, and then normalized to experimental controls.

ELISA

The concentration of secreted MMP13 in the conditioned media was determined by ELISA Kit (R\&D System, Los Angeles, CA, USA). ELISAs were performed according to the instructions of the manufacturer. Briefly, the collected condition media was added to a well coated with primary antibody, and then immunosorbented by biotinylated primary antibody at room temperature for 2 hours. The color development catalyzed by horseradish peroxidase was terminated with $2.5 \mathrm{~mol} / \mathrm{l}$ sulfuric acid and the absorption was measured at $450 \mathrm{~nm}$. The protein concentration was determined by comparing the relative absorbance of the samples with the standards.

\section{Statistical analysis}

All statistical analyses were carried out using the SPSS 17.0 statistical software package. All values are depicted as mean \pm standard deviation and are considered significant if $p<0.05$. All data were statistically analyzed using one-way ANOVA with a Bonferoni correction, followed by Fisher's Exact Test to compare two groups. Bivariate correlations were calculated by Spearman's rank correlation coefficients.

\section{Results}

Low levels of FGFR3 and high levels of miR-100 are inversely correlated in LDD

We examined the FGFR1, FGFR3 and miR-100 levels in the 21 LDD discs and 16 traumatized, non-LDD discs (NT) from the patients. We detected significantly higher levels of FGFR1 (Fig. 1A) and significantly lower levels of FGFR3 (Fig. 1B) in LDD by Western blot. We also detected significantly higher levels of miR-100 in LDD by RT-qPCR (Fig. 1C). Then we studied the correlation between FGFR1 and miR-100, and between FGFR3 and miR100, to look for a regulatory relationship. No correlation was detected between FGFR1 and miR-100 in LDD specimen (Fig. 1D, $R=0.47$; $\mathrm{p}=0.03$ ). However, a strong inverse correlation was detected between FGFR3 and miR-100 in LDD specimen (Fig. 1E, R= -0.90; p < 0.0001), suggesting a causal relationship. 


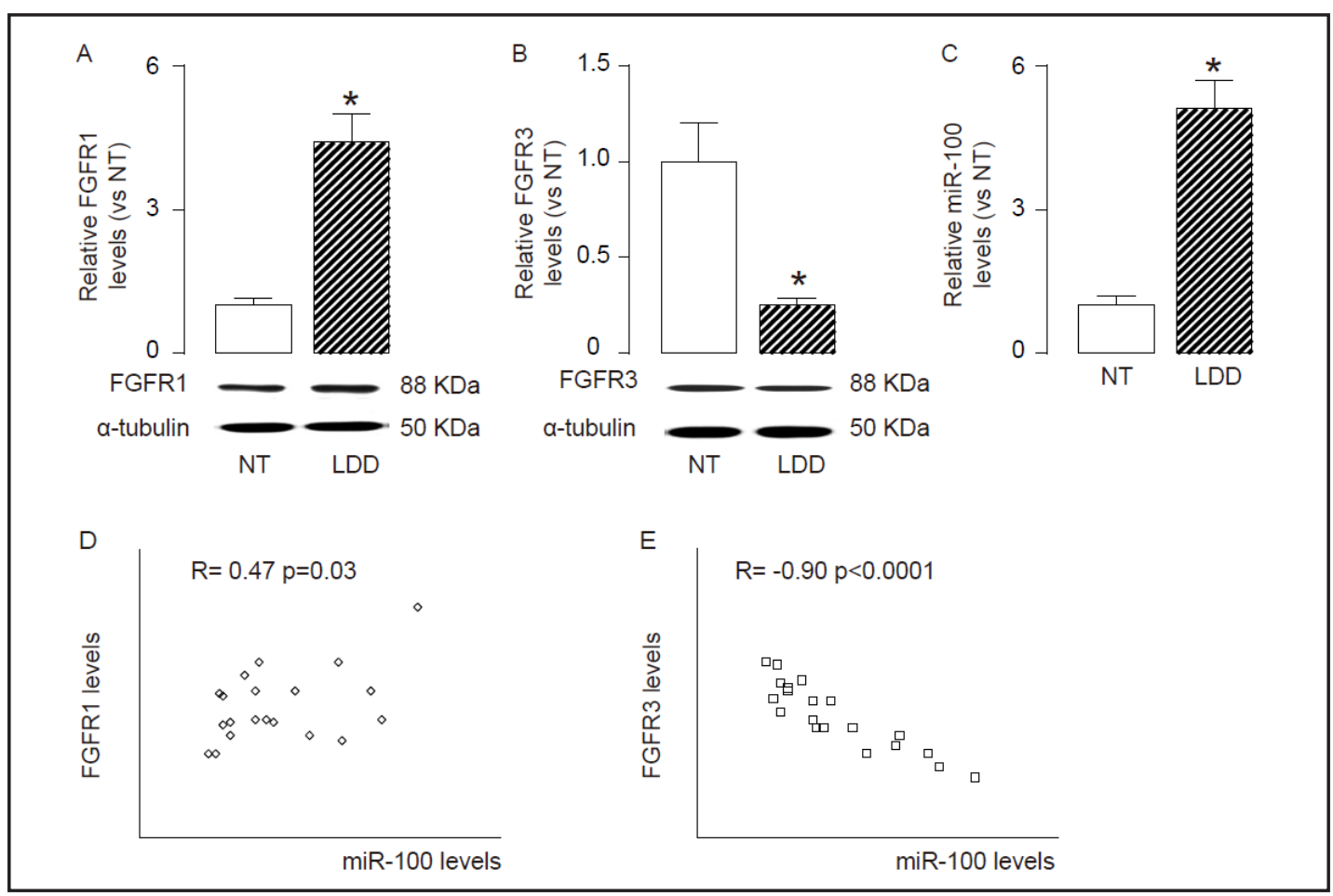

Fig. 1. Low levels of FGFR3 and high levels of miR-100 are inversely correlated in LDD. We examined the FGFR1, FGFR3 and miR-100 levels in the 21 LDD discs and 16 traumatized, non-LDD discs (NT) from the patients. (A-B) We detected significantly higher levels of FGFR1 (A) and significantly lower levels of FGFR3 (B) in LDD by Western blot. (C) We also detected significantly higher levels of miR-100 in LDD by RT-qPCR. (D) No correlation was detected between FGFR1 and miR-100 in LDD specimen ( $R=0.47 ; p=0.03)$. (E) A strong inverse correlation was detected between FGFR3 and miR-100 in LDD specimen $(\mathrm{R}=-0.90 ; \mathrm{p}<$ 0.0001). Statistics: one-way ANOVA with a Bonferoni correction, followed by Fisher's Exact Test to compare two groups.*: $\mathrm{p}<0.05$.

MiR-100 targets 3'UTR of FGFR3 to inhibit its expression

Based on our findings in the patients' samples, we performed bioinformatics analysis of FGFR3 target sequence, which shows that miR-100 binds to 3'UTR of FGFR3 mRNA at $537^{\text {th }}-544^{\text {th }}$ base site (Fig. $2 \mathrm{~A}$ ). To prove that this binding has a biological effect, we modified miR-100 levels in a human disc cell line, HNPSV. We transfected HNPSV cells with either miR-100 or antisense for miR-100 (as-miR-100). HNPSV cells were also transfected with a scramble sequence as a control (scr). Modulation of miR-100 levels in HNPSV cells was confirmed by RT-qPCR (Fig. 2B). Then, HNPSV-miR-100, HNPSV-scr and HNPSV-as-miR-100 cells were transfected with $1 \mu \mathrm{g}$ of FGFR3-3'UTR Luciferase-reporter plasmid. We found that the luciferase activities in HNPSV-as-miR-100 cells were significantly higher than the control scr, while the luciferase activities in HNPSV-miR-100 cells were significantly lower than the control scr (Fig. 2C). These data suggest that miR-100 targets 3'UTR of FGFR3 to inhibit its translation.

MiR-100 decreases FGFR3 protein but does not affect its transcription

Since FGFR3 is known to compete with FGFR1 for FGF2 binding in disc cells and FGF2/ FGFR1 signaling activates MMP13 to induce disc degradation, we were thus prompted to evaluate whether FGFR3 may be regulated by miR-100 in disc cells. We found that although the FGFR3 transcripts did not change by miR-100 levels (Fig. 3A), the protein levels of FGFR3 in miR-100-overexpressing HNPSV cells was significantly decreased, while the protein levels of FGFR3 in miR-100-depleted HNPSV cells was significantly increased (Fig. 3B). These data suggest that the translation of FGFR3 in disc cells may be regulated by miR-100. 


\section{A}

predicted binding of miR-100 to FGFR3 3'-UTR

5' CucAgagacugaAaudacggua 3'

| |||||

3'

GUGUUCAAGCCUAGAUGCCCAA 5'

FGFR3 3'-UTR (537-544)

$\operatorname{miR}-100$
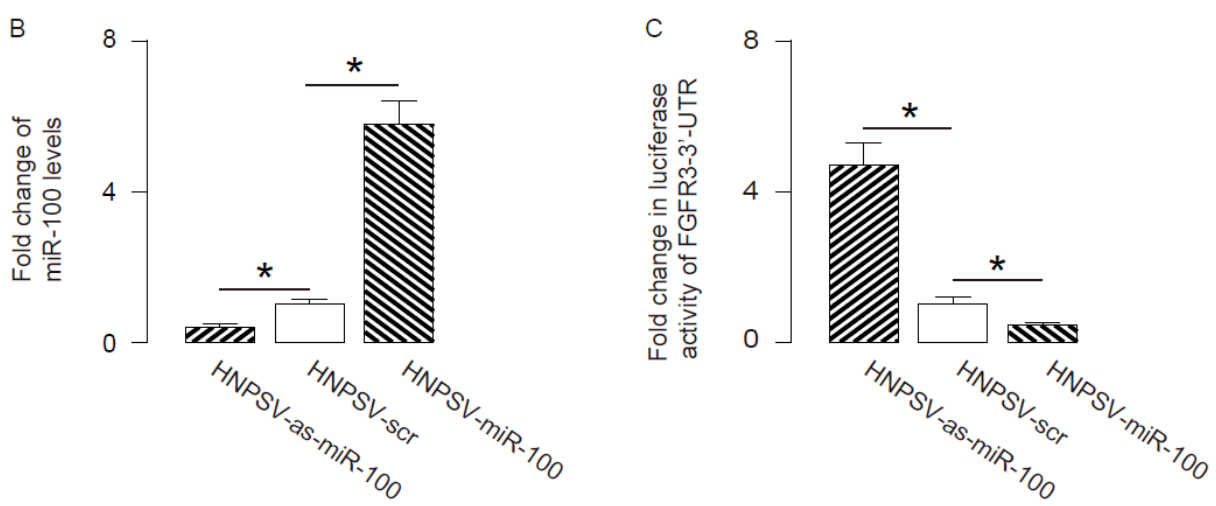

Fig. 2. MiR-100 targets 3 'UTR of FGFR3 to inhibit its expression. (A) Bioinformatics analysis of FGFR3 target sequence, showing that miR-100 binds to $3^{\prime}$ UTR of FGFR3 mRNA at $537^{\text {th }}-544^{\text {th }}$ base site. (B-C) We modified miR-100 levels in a human disc cell line, HNPSV, by transfecting HNPSV cells with either miR-100 or antisense for miR-100 (as-miR-100) or a scramble sequence as a control (scr). (B) Modulation of miR-100 levels in HNPSV cells was confirmed by RT-qPCR. (C) HNPSV-miR-100, HNPSV-scr and HNPSV-as-miR-100 cells were transfected with $1 \mu \mathrm{g}$ of FGFR3-3'UTR Luciferase-reporter plasmid. The luciferase activities were quantified. Statistics: one-way ANOVA with a Bonferoni correction, followed by Fisher's Exact Test to compare two groups.*: $\mathrm{p}<0.05$.

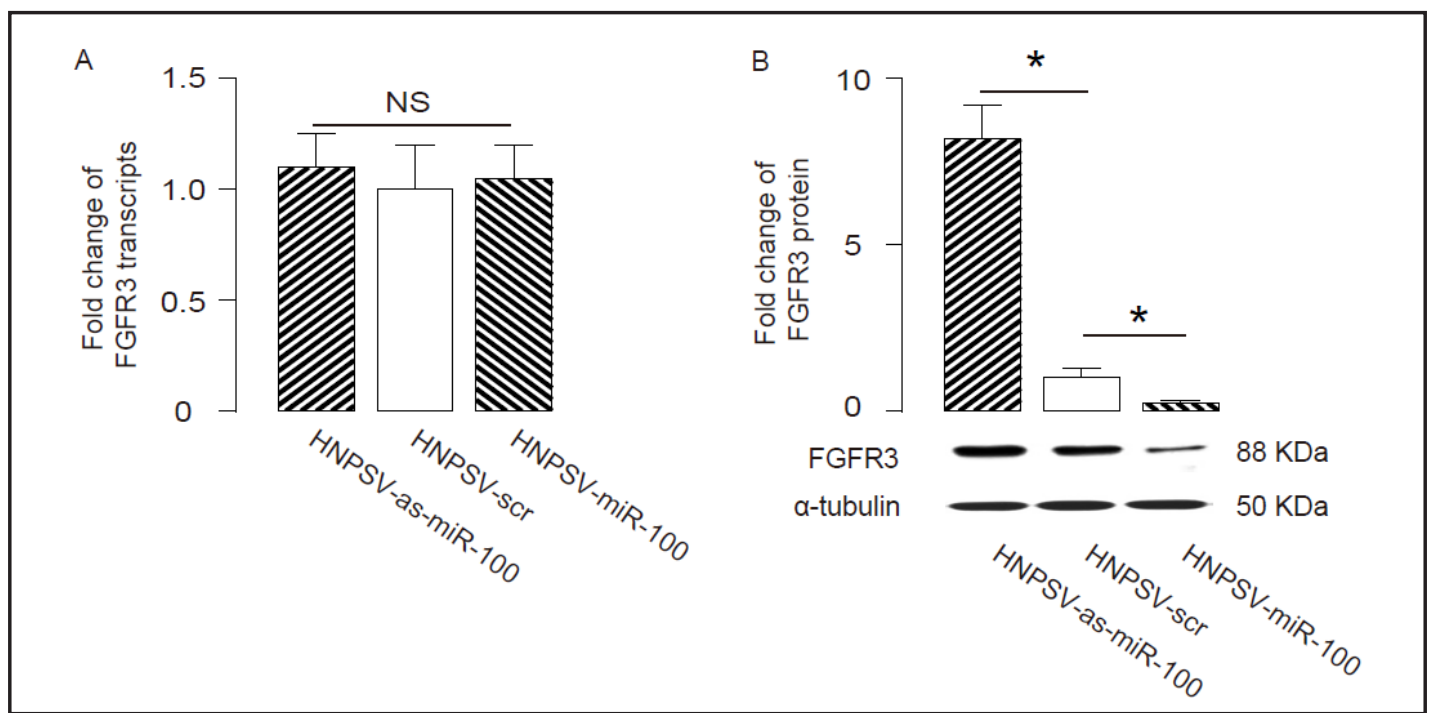

Fig. 3. MiR-100 decreases FGFR3 protein but does not affect its transcription. (A) RT-qPCR for FGFR3 in miR-100-modified HNPSV cells. (B) The Western blot for FGFR3 in miR-100-modified HNPSV cells. Statistics: one-way ANOVA with a Bonferoni correction, followed by Fisher's Exact Test to compare two groups.*: p<0.05. NS: non-significant.

MiR-100 activates MMP13 in disc cells

Then we examined whether the effects of miR-100 on FGFR3 may result in changes in MMP13 in disc cells. We found that miR-100-overexpression in HNPSV cells increased 
A

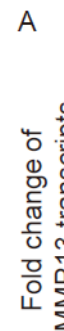

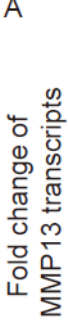
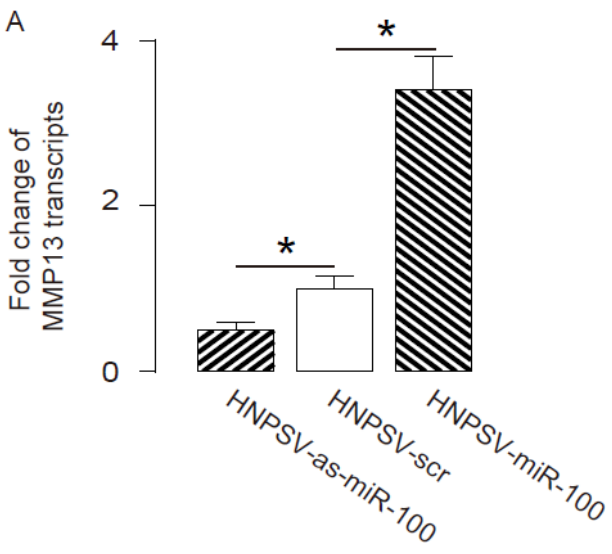

C

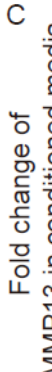

*

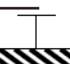

ה্র

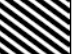

$\sim 2$
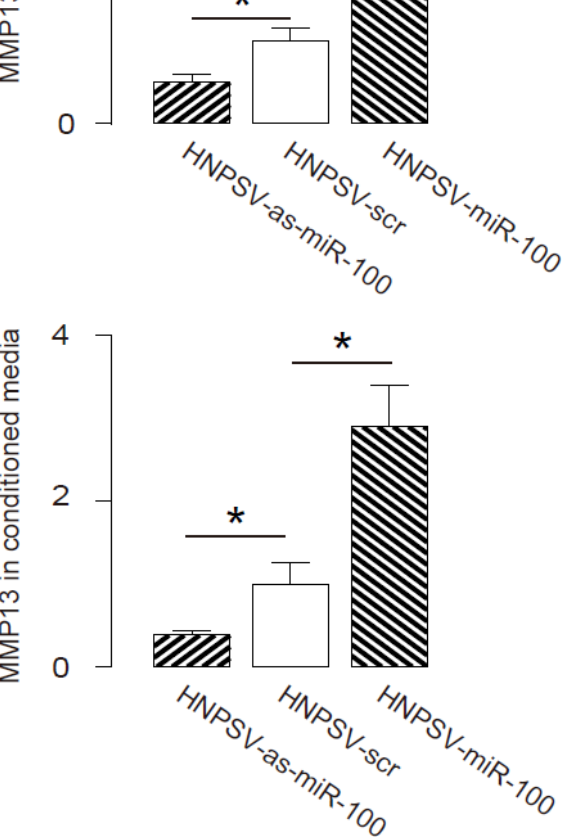

B

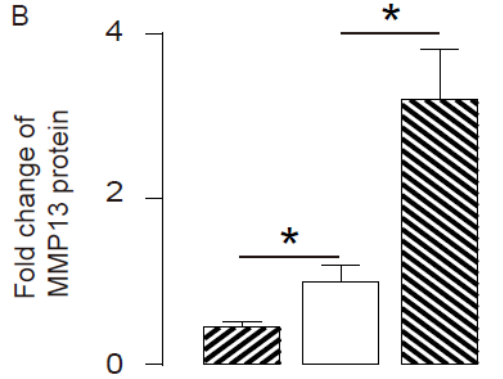

MMP13

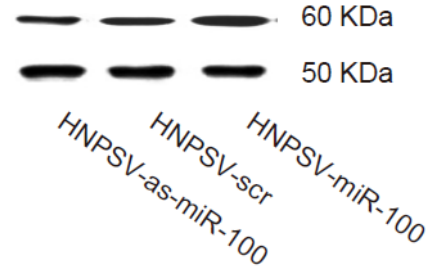

Fig. 4. MiR-100 activates MMP13 in disc cells. (A-C) We found that miR-100-overexpression in HNPSV cells increased MMP13 levels by RT-qPCR (A), by Western blot (B), and by secreted protein in the conditioned media (C). On the other hand, miR-100-depletion in HNPSV cells decreased MMP13 levels by RT-qPCR (A), by Western blot (B), and by secreted protein in the conditioned media (C). Statistics: one-way ANOVA with a Bonferoni correction, followed by Fisher's Exact Test to compare two groups.*: $\mathrm{p}<0.05$.

Fig. 5. Schematic of the model. MiR-100 promotes disc degradation through FGFR3 suppression, which may activate MMP13 via imbalance of FGFR1 and FGFR3 levels.

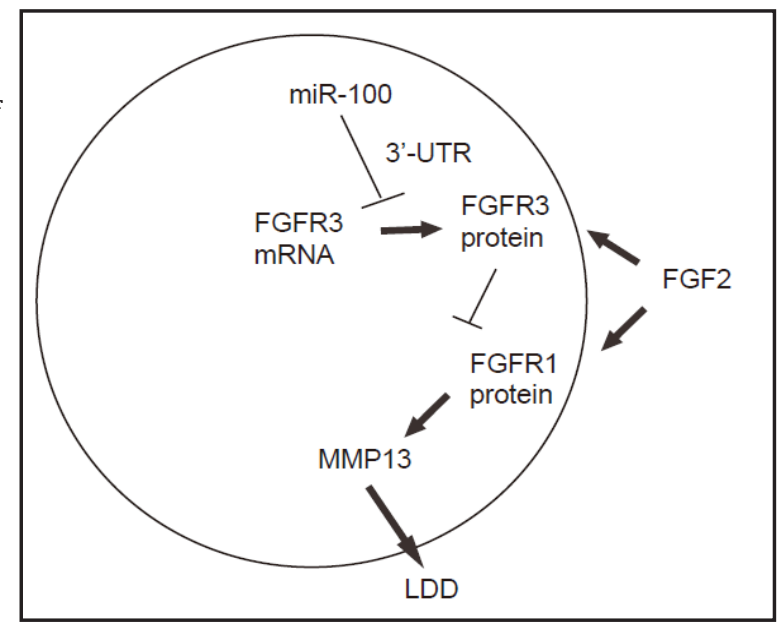

MMP13 levels by RT-qPCR (Fig. 4A), by Western blot (Fig. 4B), and by secreted protein in the conditioned media (Fig. 4C). On the other hand, miR-100-depletion in HNPSV cells decreased MMP13 levels by RT-qPCR (Fig. 4A), by Western blot (Fig. 4B), and by secreted protein in the conditioned media (Fig. 4C). Together, our study demonstrates that miR-100 promotes disc degradation through FGFR3 suppression (Fig. 5). 


\section{Cellular Physiology Cell Physiol Biochem 2015;36:2229-2236 \begin{tabular}{ll|l} 
DOI: 10.1159/000430187 & $\begin{array}{l}\text { O 2015 S. Karger AG, Basel } \\
\text { www.karger.com/cpb }\end{array}$ \\
\hline
\end{tabular} \\ Yan/Yu/Zhang/Hou: MiR-100 Promotes LDD through FGFR3}

\section{Discussion}

Previous efforts have resulted in identification of a critical signal transduction pathway of FGF2-stimulated, MMP13-mediated development of LDD. Indeed, FGF2 acts as an anabolic mediator of cartilage homeostasis due to its mitogenic capacity, whereas the mitogenic effect of FGF2 in human discs may be a pathologic sign of degeneration rather than regeneration. The increase in cell proliferation mediated by FGF2 may stimulate an upregulation of collagen I, and result in a decrease in collagen II:I ratio. Moreover, the potent mitogenic effect of FGF2 in disc cells has previously been correlated with FGF receptor activation. In disc cells, among all 4 FGFRs, only FGFR1 and FGFR3 are expressed. However, FGF2-activated FGFR1 or FGFR3 may initiate different signaling pathways. In the growth plate, FGFR1 and FGFR3 have significant yet opposite roles in cell homeostasis. Binding of FGF2 to FGFR1 increases proliferation of disc cells, whereas binding of FGF2 to FGFR3 inhibits proliferation and therefore promotes disc cell differentiation. Thus, in the absence of signaling from FGFR3, a compensatory increase in interaction between FGF2 and FGFR1 may result in degradative effects with increased MMP-13 expression and increased cleavage products from type II collagen. The evidence of involvement of other MMPs in the development of LDD is much lacking.

Here, we detected strong inverse correlation between FGFR3 and miR-100, but not between FGFR1 and LDD, in LDD specimen, suggesting presence of a regulatory relationship. Moreover, this hypothesis was proved by luciferase reporter assay, in which we identified a binding site of miR-100 at 3'UTR on FGFR3 mRNA, which is functional. We failed to detect any binding sites on 3'UTR on FGFR1 mRNA by miR-100. These data are consistent with what we found in LDD specimen, and demonstrate that FGFR3 translational controls in disc cells may be regulated by miR-100.

We thus examined whether miR-100 may inhibit the expression of FGFR3. We either overexpressed or inhibited miR-100 expression in disc cells, which decreased or increased the protein levels of FGFR3, respectively, without affecting FGFR3 transcript levels. Taken together, these findings strongly demonstrate a critical role of miR-100 in regulating FGFR3 translation, rather than in regulating the degradation of FGFR3 mRNA. Moreover, the modulated FGFR3 levels by miR-100 resulted in regulation of MMP13 activation at both transcription and translation levels. These data are consistent with previous studies and suggest that miR-100/FGFR3 axis may be a potent therapeutic target for inhibiting the development of LDD. In future, experiments should be designed to determine why miR-100 has other targets in the LDD pathological process.

\section{Disclosure Statement}

The authors have declared that no competing interests exist.

\section{Reference}

1 Wang SZ, Rui YF, Lu J, Wang C: Cell and molecular biology of intervertebral disc degeneration: Current understanding and implications for potential therapeutic strategies. Cell Prolif 2014;47:381-390.

2 Wang HQ, Samartzis D: Clarifying the nomenclature of intervertebral disc degeneration and displacement: From bench to bedside. Int J Clin Exp Pathol 2014;7:1293-1298.

3 Risbud MV, Shapiro IM: Role of cytokines in intervertebral disc degeneration: Pain and disc content. Nat Rev Rheumatol 2014;10:44-56.

4 Feng C, Liu H, Yang Y, Huang B, Zhou Y: Growth and differentiation factor-5 contributes to the structural and functional maintenance of the intervertebral disc. Cell Physiol Biochem 2015;35:1-16. 


\section{Cellular Physiology Cell Physiol Biochem 2015;36:2229-2236 \begin{tabular}{l|l} 
and Biochemistry Published online: July 24, 2015 & $\begin{array}{l}\text { DOI: 10.1159/000430187 2015 S. Karger AG, Basel } \\
\text { www.karger.com/cpb }\end{array}$ \\
\hline
\end{tabular} \\ Yan/Yu/Zhang/Hou: MiR-100 Promotes LDD through FGFR3}

$5 \quad$ Chen JW, Ni BB, Li B, Yang YH, Jiang SD, Jiang LS: The responses of autophagy and apoptosis to oxidative stress in nucleus pulposus cells: Implications for disc degeneration. Cell Physiol Biochem 2014;34:11751189.

6 Diederichs S, Zachert K, Raiss P, Richter W: Regulating chondrogenesis of human mesenchymal stromal cells with a retinoic acid receptor-beta inhibitor: Differential sensitivity of chondral versus osteochondral development. Cell Physiol Biochem 2014;33:1607-1619.

7 Zheng S, Zhong ZM, Qin S, Chen GX, Wu Q, Zeng JH, Ye WB, Li W, Yuan K, Yao L, Chen JT: Advanced oxidation protein products induce inflammatory response in fibroblast-like synoviocytes through nadph oxidase -dependent activation of nf-kappab. Cell Physiol Biochem 2013;32:972-985.

8 Tan MY, Mu XY, Liu B, Wang Y, Bao ED, Qiu JX, Fan Y: Sumo-specific protease 2 suppresses cell migration and invasion through inhibiting the expression of mmp13 in bladder cancer cells. Cell Physiol Biochem 2013;32:542-548.

9 Stockl S, Gottl C, Grifka J, Grassel S: Sox9 modulates proliferation and expression of osteogenic markers of adipose-derived stem cells (asc). Cell Physiol Biochem 2013;31:703-717.

10 Fosang AJ, Last K, Knauper V, Murphy G, Neame PJ: Degradation of cartilage aggrecan by collagenase-3 (mmp-13). FEBS Lett 1996;380:17-20.

11 Huang GF, Zou J, Shi J, Zhang DY, Pen HF, Zhang Q, Gao Y, Wang BY: The effect of electroacupuncture on the extracellular matrix synthesis and degradation in a rabbit model of disc degeneration. Evidence-based complementary and alternative medicine : eCAM 2014;2014:731395.

12 Hegewald AA, Zouhair S, Endres M, Cabraja M, Woiciechowsky C, Thome C, Kaps C: Towards biological anulus repair: Tgf-beta3, fgf- 2 and human serum support matrix formation by human anulus fibrosus cells. Tissue \& cell 2013;45:68-76.

13 Yan D, Chen D, Im HJ: Fibroblast growth factor-2 promotes catabolism via fgfr1-ras-raf-mek1/2-erk1/2 axis that coordinates with the pkcdelta pathway in human articular chondrocytes. J Cell Biochem 2012;113:2856-2865.

14 Li X, An HS, Ellman M, Phillips F, Thonar EJ, Park DK, Udayakumar RK, Im HJ: Action of fibroblast growth factor-2 on the intervertebral disc. Arthritis Res Ther 2008;10:R48.

15 Wang J, Liu W, Peng Y, Li W, Cheng T, Gao C, Mo L, Zhao Z, Chang L: Synergetic effect of alpha-lipoic acid with keratinocyte growth factor on protecting alveolar epithelial type ii cells of rat fetus from hyperoxia -induced injury. Cell Physiol Biochem 2014;33:953-966.

16 Ornostay A, Cowie AM, Hindle M, Baker CJ, Martyniuk CJ: Classifying chemical mode of action using gene networks and machine learning: A case study with the herbicide linuron. Comp Biochem Physiol Part D Genomics Proteomics 2013;8:263-274.

17 Matsubara Y, Aoki M, Endo T, Sato K: Characterization of the expression profiles of adipogenesis-related factors, znf423, klfs and fgf10, during preadipocyte differentiation and abdominal adipose tissue development in chickens. Comp Biochem Physiol B Biochem Mol Biol 2013;165:189-195.

18 Ellman MB, Yan D, Ahmadinia K, Chen D, An HS, Im HJ: Fibroblast growth factor control of cartilage homeostasis. J Cell Biochem 2013;114:735-742.

19 Ellman MB, An HS, Muddasani P, Im HJ: Biological impact of the fibroblast growth factor family on articular cartilage and intervertebral disc homeostasis. Gene 2008;420:82-89.

20 Sicard F, Gayral M, Lulka H, Buscail L, Cordelier P: Targeting mir-21 for the therapy of pancreatic cancer. Mol Ther 2013;21:986-994.

21 Tavano F, di Mola FF, Piepoli A, Panza A, Copetti M, Burbaci FP, Latiano T, Pellegrini F, Maiello E, Andriulli A, di Sebastiano P: Changes in mir-143 and mir-21 expression and clinicopathological correlations in pancreatic cancers. Pancreas 2012;41:1280-1284.

22 Ali S, Ahmad A, Banerjee S, Padhye S, Dominiak K, Schaffert JM, Wang Z, Philip PA, Sarkar FH: Gemcitabine sensitivity can be induced in pancreatic cancer cells through modulation of mir-200 and mir-21 expression by curcumin or its analogue cdf. Cancer Res 2010;70:3606-3617.

23 Sakai D, Mochida J, Yamamoto Y, Toh E, Iwashina T, Miyazaki T, Inokuchi S, Ando K, Hotta T: Immortalization of human nucleus pulposus cells by a recombinant sv40 adenovirus vector: Establishment of a novel cell line for the study of human nucleus pulposus cells. Spine 2004;29:1515-1523. 